\title{
Neurocognitive effects of repeated ketamine infusion treatments in patients with treatment resistant depression: A retrospective chart review
}

Danika Dai

McLean Hospital

Courtney Miller

McLean Hospital

Violeta Valdivia

McLean Hospital

Brian Boyle

McLean Hospital

Shuang Li ( $\sim$ Shuang.Li@MGH.HARVARD.EDU)

McLean Hospital

Steve Seiner

McLean Hospital

Robert Meisner

McLean Hospital

\section{Research Article}

Keywords: ketamine, repeated intravenous infusions, neurocognition, treatment-resistant depression

Posted Date: November 8th, 2021

DOI: https://doi.org/10.21203/rs.3.rs-1017633/v1

License: (c) (i) This work is licensed under a Creative Commons Attribution 4.0 International License.

Read Full License

Version of Record: A version of this preprint was published at BMC Psychiatry on February 22nd, 2022. See the published version at https://doi.org/10.1186/s12888-022-03789-3. 


\section{Abstract}

\section{Background}

Ketamine has emerged as a rapid-acting antidepressant in treatment-resistant depression (TRD) increasingly used in non-research, clinical settings. Few studies, however, have examined neurocognitive effects of repeated racemic ketamine infusion treatments in patients with TRD. In an effort to identify potential effects after serial infusions, we conducted a retrospective chart review to identify statistically significant changes in cognition in patient undergoing serial intravenous infusions; concomitantly, we examined baseline cognition as potential predictor of anti-depressant potential.

Methods

Twenty-two patients with TRD were examined after they finished the induction phase of 8-10 repeated intravenous ketamine infusions and completed the assessments of their depressive symptoms (measured by the 16-item Quick Inventory of Depressive Symptomatology-Self Report Scale: QIDS-SR16) and cognitive function (measured by the Montreal Cognitive Assessment: MoCA) before the first and the last ketamine treatments.

Results

Repeated ketamine infusions administered through an escalating dose protocol with 8-10 infusion sessions produced a $47.2 \%$ reduction response in depression; there was no evidence of impairment as reflected in MoCA testing. There was a moderate association between baseline cognition and antidepressant response with a pearson correlation of 0.453 .

Conclusion

In this naturalistic sample of patients with TRD in our clinical service, repeated ketamine infusions significantly decreased depression symptoms without impairing cognitive performance. The baseline cognition may positively predict antidepressant responses of repeated ketamine treatment.

\section{Background}

Major depressive disorder (MDD) is a common and disabling mental condition that affects up to one in five adults in the United States during their lifetime, and more than 300 million people worldwide at any given time, according to the World Health Organization (Hasin et al., 2018; World Health Organization, 2017). Furthermore, up to one-third of patients are considered to have treatment-resistant depression (TRD), defined as an insufficient response to two more adequate trials of antidepressant medications [3].

Ketamine, an N-methyl-D-aspartate receptor antagonist, was demonstrated to have a rapid antidepressant action in a randomized double-blind trial for the first time in 2000 [4]. Since then, emerging studies have indicated rapid and robust antidepressant effects of ketamine in adults with TRD (Andrade, 2019; 
Domany et al., 2019; Fava et al., 2020b; Marcantoni et al., 2020; Wilkinson and Sanacora, 2016). A single subanesthetic $(0.5 \mathrm{mg} / \mathrm{kg})$ dose infusion of intravenous (IV) ketamine has rapid-acting and robust antidepressant effects in at least $50 \%$ of patients, however, the effects dissipate by day 10 to day 14 [1014]. Patients not responsive to a single infusion of ketamine may improve with subsequent infusions, and improvement following a single infusion can be sustained by subsequent infusions [15-17].

Cognition plays a key role in recovery and functional outcomes in MDD [18]. Given known harmful effects of ketamine in cognition when used in cohorts struggling with ketamine use disorders, there has remained concern that serial administration of low dose ketamine could have the potential to negatively affect cognition. Human results of acute ketamine use on memory are mixed [19]. Results of studies on chronic ketamine use have suggested that individuals who had taken high doses of ketamine frequently or abused it for a long period of time could be more prone to experiencing serious neurocognitive impairment [20-22]. Furthermore, a single infusion of ketamine $(0.4 \mathrm{or} 0.8 \mathrm{mg} / \mathrm{kg})$ induced dosedependently impaired episodic and working memory and slowed semantic processing, recognition memory, and procedural learning, and infusion of analgesic doses $(8-20 \mathrm{mg} / \mathrm{h})$ in healthy volunteers was shown to produce significant deficits in cognition $[23,24]$. On the other hand, Ning's group noted no deterioration in cognitive function from six ketamine infusions at $0.5 \mathrm{mg} / \mathrm{kg}$ over 12 days $[25,26]$. Instead, a single infusion of $0.5 \mathrm{mg} / \mathrm{kg}$ in TRD patients was seen to be slightly beneficial in attention and response control [27].

As concerns regarding ketamine and cognition have evolved, concomitant curiosity regarding the predictive value of baseline neurocognitive function has emerged $[26,28,29]$. Murrough et al (2014) have suggested individuals with TRD performing with lower neurocognitive function at baseline were more likely to obtain a positive antidepressant response from a single ketamine infusion. On the other hand, a study conducted be Bönke's group indicated no significant correlation between baseline cognitive performance and a change in symptom severity, nor a correlation between a change in cognitive performance and antidepressant responses from six series ketamine infusion treatments [30].

In 2018, we began to treat patients with TRD by ketamine infusion in the translational Ketamine Service at McLean Hospital. Over the years, we have refined a serial infusion ketamine protocol based on the evolving literature and our clinical experience. The protocol includes an induction phase and either a booster or maintenance phase. The induction phase consists of 8-10 treatments on a twice-weekly schedule with the IV ketamine does initiated at $0.5 \mathrm{mg} / \mathrm{kg}$ over 40 minutes; thereafter, titration to response was permitted in a conservative fashion at the discretion of the ketamine team with the maximum dose up to $1.0 \mathrm{mg} / \mathrm{kg}$. After the induction phase, if the patient achieved a substantial response, patients had the option of completing the phase with a brief taper series. Thereafter, patients were instructed to return for booster infusions (generally between 1 and 3 infusions) if depression symptoms returned.

In this real-world context, the investigation below seeks to 1) report the neurocognitive effects of repeated ketamine infusions in patients with TRD who have finished the induction phase through a retrospective 
chart review and 2) examine whether there is an association between cognition at baseline and antidepressant effects induced by repeated ketamine infusion.

\section{Methods}

\section{Clinical procedure}

Ketamine treatment was offered to patients with severe and refractory MDD with at least two or more adequate anti-depressant treatment failures, while patients with a history of psychosis, current substance use disorder, or uncontrolled medical illness, were not eligible for ketamine treatment. After psychiatric consultation and medical assessment, patients who were appropriate for ketamine treatment reviewed and signed a consent for ketamine treatment that emphasized that ketamine was not approved by the U.S. FDA for any psychiatric indication, and was provided off-label for depression, in addition to potential risks and benefits. At each visit, patients were evaluated and monitored by a staff psychiatrist, a nurse, and an anesthesiologist.

Depression symptom severity was evaluated with the 16-item Quick Inventory of Depressive Symptomatology-Self Report scale (QIDS-SR16) that is scored on a scale of 0 to 27, with 0 representing a complete absence of depressive symptoms and 27 representing the most severe symptoms [31]. The QIDS-SR16 was administered before at baseline and every subsequent visit.

Cognition was evaluated with the Montreal Cognitive Assessment (MoCA) [32]. The 30-item MoCA, a brief cognitive screening tool for cognitive impairment, is recognized as a sensitive measure of cognitive function that can capture declines in cognition over repeated administrations. The MoCA is scaled from 1 to 30, with higher MoCA scores indicating better cognitive function; a MoCA score <26 indicates impaired cognitive function [32]. The MoCA includes six cognitive domains, including visuospatial abilities; language; combined attention, concentration, and working memory; executive function; short-term memory recall; and orientation to time and place, although subsequent consensus has emerged that the total score is most meaningful. The MoCA test was provided by clinic staff trained in its administration before the first treatment and the last treatment of induction.

Eligible participants for ketamine infusions received an acute induction course consisting of 8-10 infusions twice weekly over 4-5 weeks. Most participants received the first dose of $0.5 \mathrm{mg} / \mathrm{kg}$ of ketamine with possible dose escalation up to $1.0 \mathrm{mg} / \mathrm{kg}$ contingent on patient tolerability to the index dose and patient's response. In terms of dosing strategy, there is currently no established consistent and optimal dose of intravenous ketamine for TRD [33]. Nevertheless, small, randomized trials that compared different doses of ketamine suggest that generally, the preferred dose may be $0.5 \mathrm{mg} / \mathrm{kg}$ of body weight [34]. However, dose adjustments may be appropriate for specific patients [35]. A dose of up to $1 \mathrm{mg} / \mathrm{kg}$ may be suitable for patients not responsive to $0.5 \mathrm{mg} / \mathrm{kg}$ [15]. In terms of frequency, in most randomized trials, the drug was given only one time and the benefit appeared to diminish over the following week. We have been using a twice-weekly schedule, consistent with the finding from a study that indicated infused 
ketamine twice-weekly or thrice-weekly for up to six weeks led to an improvement of depression with the two dosing frequencies comparable [36]. All infusions were administered over a period of 40-45 min, and participants were monitored at the clinic for up to two hours following treatment.

During the treatments, other pharmacological and psychotherapeutic treatments were continued as part of the usual regimen. In the procedure, patients who experienced transient dissociative symptoms or anxiety during the infusions could receive either intravenous infusion or $1 \mathrm{mg}$ of oral lorazepam to improve the tolerability of the infusion. Patients who experienced nausea could receive intravenous or oral ondansetron. Blood pressure, heart rates, oxygen saturation, and respiration rates were monitored at regular intervals ( 5 mins, then $10 \mathrm{~min}$, then each $15 \mathrm{~min}$ ) during the infusion and for 30 minutes afterward, with contingency planning for additional monitoring as clinically appropriate. Patients with a clinically significant increase in blood pressure could receive intravenous or oral labetalol. Criteria for discharge readiness included a return to baseline mental status, absence of gait disturbance and nausea, and normal blood pressure. Any administration required the patient to be discharged to the care of an adult escort, and driving was not permitted in the evening post-administration until the following day.

\section{Statistical Analyses}

In the present report, patients with MDD who started ketamine treatment from July 2018 to May 2021 were included. This retrospective data analysis was approved by the Institutional Review Board (IRB) of Mass General Brigham. Patients were included in the final analyses if they completed the induction treatment phase (8-10 sessions) and had completed MoCA and QIDS-SR16 questionnaires measured at baseline and on the morning of the last treatment.

A two-tailed paired t-test was used to compare changes in metric scores between baseline and posttreatment. A logistic regression analysis was used to determine whether initial cognition scores measured by MoCA predict antidepressant response measured by reduction of QIDS-SR16 between baseline and post-treatment from repeated ketamine intravenous infusions. Percentage of reduction of QIDS-SR16 was calculated using the following formula: (baseline QIDS-SR16-post treatment QIDS-SR16)/post treatment QIDS-SR16*100. All analyses were performed using IBM SPSS version 21 and a $p$-value of $<0.05$ was considered statistically significant.

\section{Results}

\section{Patient clinical and demographic characteristics}

From July 2018 to May 2021, seventy patients finished the induction phase for 8-10 intravenous ketamine treatments. Among those patients, twenty-two patients completed their depressive symptoms measured by QIDS-SR16 and neurocognitive function measured by MoCA before the first and last treatment. Table 1, summarized below, presents the demographic characteristics of these patients with TRD. 
Table 1

Clinical and demographic characteristics of these patients.

\begin{tabular}{|c|c|}
\hline Characteristics & Patients for ketamine infusion treatment $(n=22)$ \\
\hline Age in years (mean+SD) & $37.86 \pm 16.95$ \\
\hline BMI (mean+SD) & $26.08+6.12$ \\
\hline Gender: Female, n (\%) & $14(63.64)$ \\
\hline \multicolumn{2}{|l|}{ Marital status, n (\%) } \\
\hline Never married/divorced & $10(45.45)$ \\
\hline Married/partner & $11(50.00)$ \\
\hline Unknown & $1(4.55)$ \\
\hline \multicolumn{2}{|l|}{ Race, n (\%) } \\
\hline White & $19(86.36)$ \\
\hline Black & $0(0)$ \\
\hline Hispanic & $0(0)$ \\
\hline Asian & $2(9.09)$ \\
\hline Unknown & $1(4.55)$ \\
\hline \multicolumn{2}{|l|}{ Educations completed, n (\%) } \\
\hline Grade $6-12$ or graduated high school & $6(27.27)$ \\
\hline Graduated 4-year college & $10(45.45)$ \\
\hline Graduate/professional degree & $6(27.27)$ \\
\hline Unknown & $0(0)$ \\
\hline \multicolumn{2}{|l|}{ Current employment status, n (\%) } \\
\hline Full-time & $8(36.36)$ \\
\hline Part-time & $1(4.55)$ \\
\hline On leave & $4(18.18)$ \\
\hline Retired & $1(4.55)$ \\
\hline Unemployed & $5(22.73)$ \\
\hline Student & $3(13.64)$ \\
\hline \multicolumn{2}{|l|}{ Hx of Substance use disorder, $n(\%)$} \\
\hline EtOH use & $3(13.64)$ \\
\hline
\end{tabular}




\begin{tabular}{|ll|}
\hline Characteristics & Patients for ketamine infusion treatment $(\mathbf{n}=\mathbf{2 2})$ \\
\hline THC & $7(31.82)$ \\
\hline Illicit substance use & $3(13.64)$ \\
\hline Current concomitant psychiatric disorder, $\mathbf{n}(\%)$ & \\
\hline Anxiety & $10(45.45)$ \\
\hline PTSD & $5(22.71)$ \\
\hline OCD & $2(9.10)$ \\
\hline ADHD & $4(18.18)$ \\
\hline Concomitant medications, $\mathbf{n}(\%)$ & \\
\hline Antidepressant drugs & 100 \\
\hline Mood stabilizer & $8(36.36)$ \\
\hline Antipsychotics & $10(45.45)$ \\
\hline Stimulants & $6(27.27)$ \\
\hline Benzodiazepines & $10(45.45)$ \\
\hline History of suicidal attempts, $\mathbf{n}(\%)$ & $6(27.27)$ \\
\hline Treatments history of ECT, $\mathbf{n}(\%)$ & $8(36.36)$ \\
\hline Treatments history of TMS, $\mathbf{n}(\%)$ & $7(31.82)$ \\
\hline
\end{tabular}

The effect of repeated ketamine infusions on cognition measured by MoCA

As shown in Figure 1, the average MoCA score at baseline was 26.77 out of 30 . The average MoCA score measured at the last treatment was 26.68 out of 30 . There was no significant difference between baseline and treatment tested by the paired t-test $(p>0.05)$

The effect of repeated ketamine infusions on depression measured by QIDS-SR16

As shown in Figure 2, patients who have completed the induction phase after repeated ketamine infusions demonstrated significant improvement in depression measured by QIDS-SR16. There was a significant reduction (47.17\%) of QIDS-SR16 measured at the last treatment compared to baseline (mean+SE: from $16.77 \pm 0.92$ at baseline down to $8.86 \pm 1.06$, two-tail paired $t$-test $p<0.001$ ).

Baseline cognition measured by MoCA predicts ketamine's antidepressant response measured by QIDSSR16 
A linear regression model was used to analyze the correlation between baseline cognition and antidepressant response after repeated ketamine infusions treatment. As shown in Figure 3 and Table 2, the baseline cognition measured by MoCA significantly correlates to antidepressant response measured by QIDS-SR16 reduction, although its effect was moderate with a Pearson correlation of 0.453 $(P=0.034<0.05)$.

Table 2

Linear regression analysis of MoCA score at baseline and reduction of QIDS-SR16 from baseline.

\begin{tabular}{|lll|}
\hline Variables & MoCA score & \\
\hline & (Pearson correlation) & Sig $P(2$-tailed) \\
\hline Reduction of QIDS-SR16 from baseline (\%) & $0.453^{*}$ & 0.034 \\
\hline *. Correlation is significant at the 0.05 level (2-tailed). \\
\hline
\end{tabular}

\section{Discussion}

We first examined the antidepressant effects of repeated 8-10 ketamine infusions at escalating dose protocol at our clinic. The results from this study suggest that patients with TRD who have completed the induction phase have demonstrated significant improvement in their depression, with a $47.2 \%$ reduction of their QIDS-SR16 score compared to their baseline. These results are consistent with other ketamine studies in similar clinical settings with non-randomized patients. Those studies indicate a response rate from $45-50 \%$ from either 6-9 infusions or 4-infusion protocol with $0.5 \mathrm{mg} / \mathrm{kg}$ of repeated intravenous ketamine treatments [30,37]. In most randomized controlled trials, the response rate to intravenous ketamine ranged from $30-70 \%$ on day $1[8,38,39]$.

Secondly, we examined the effects of repeated escalating doses of ketamine intravenous treatment on the cognitive function. To the best of our knowledge, this is one of few studies that have investigated the effects of repeated escalating doses of ketamine intravenous treatment on the cognitive function of patients with TRD and compared the relationship of baseline cognitive function with antidepressant response in the clinical practice setting.

The fear of cognitive impairment can be a major barrier to repeated ketamine treatment in clinical practice. Studies have indicated that a cumulative dose of ketamine leads to neurocognitive impairments, decreased hippocampal function, and BDNF, suggesting potential dose-, frequency- and durationdependent effects on cognition with ketamine [21, 40,41]. Higher doses, higher frequencies, or longer durations of ketamine may cause more serious neurocognitive problems. In our clinical practice, we have demonstrated that repeated ketamine intravenous treatment at escalating dose protocol with 8-10 infusion sessions did not impair cognitive performance. There was no significant change in MoCA scores between post-infusions and baseline. Compared to previous studies, which have indicated that a single ketamine infusion at $0.5 \mathrm{mg} / \mathrm{kg}$ improved specific cognition as measured by the go/no-go task and six 
ketamine infusions at $0.5 \mathrm{mg} / \mathrm{kg}$ improved verbal learning and speed of processing, we did not find that repeated ketamine treatment improved cognitive function as measured by MoCA. Possible explanations for this discrepancy could be due to a different dosing schedule and different cognitive tests [26, 27]. In our clinical procedure, the starting dose was $0.5 \mathrm{mg} / \mathrm{kg}$ of ketamine, with a possible dose escalation of up to $1.0 \mathrm{mg} / \mathrm{kg}$ contingent on patient tolerability to the index dose and patient's response. In addition, we administered the MoCA test post 8-12 sessions, instead of a single session (Chen et al., 2018) or 6 sessions (Zhou et al., 2018). Finally, the MoCA test could be different compared to other cognitive tests in terms of its sensitivity.

Regarding the relationship between baseline cognitive function and antidepressant response from repeated intravenous ketamine infusion treatments, we found that the baseline cognition measured by MoCA positively correlates to antidepressant response measured by QIDS-SR16 reduction. A higher MoCA score predicts a better antidepressant outcome. Although the study from Bönke's group (2020) indicated no significant correlation between baseline cognitive performance and a change in symptoms severity, our findings are consistent with the finding that individuals with better visual learning at baseline were likely to obtain antidepressant response over six ketamine infusions [26].

This study has several limitations. First, as this is a retrospective chart review, patients were followed naturalistically without randomization nor a well-controlled group. They could continue or change antidepressants and psychotherapy regimen according to recommendations from their treating psychiatrist. Potential confounding effects due to natural changes or other treatments, including changes in antidepressants and psychotherapy, cannot be ruled out. Second, in our clinical practice, there was no fixed dosing schedule. We chose the first dose of ketamine intravenous at $0.5 \mathrm{mg} / \mathrm{kg}$, with a possible escalating dose of up to $1.0 \mathrm{mg} / \mathrm{kg}$ depending on response and tolerability. The sessions for the induction phase could range from $8-10$, with the majority being 8 sessions. Third, the high drop-out rate observed in our sample may have been influenced by the cost of ketamine infusions, since this treatment is not currently covered by most insurance companies. Due to their unpredictable busy schedules, more than $50 \%$ of patients who had completed the induction phase have either refused to finish the posttreatment MoCA assessment or were not offered the assessment by staff members.

\section{Conclusion}

In conclusion, in this naturalistic sample of patients with TRD, repeated ketamine intravenous infusions significantly improves depression, with $47.2 \%$ of reduction of their depression symptoms. Repeated ketamine intravenous treatment at escalating dose protocol with 8-10 infusion sessions did not impair cognitive performance. There was a moderate association between baseline cognition as measured by the MoCA and antidepressant response from intravenous ketamine treatment.

\section{Abbreviations}

TRD 
Treatment-resistant depression

MDD

Major depressive disorder

QIDS-SR16

16-item Quick Inventory of Depressive Symptomatology-Self Report Scale

MoCA

Montreal Cognitive Assessment

IV

Intravenous

\section{Declarations}

\section{Funding}

Not applicable

\section{Availability of data and materials}

The data cannot be shared due to confidentiality issues. Shuang Li, (MD, PhD. at Psychiatric Neurotherapeutics Program, McLean Hospital, 115 Mill St, Belmont, MA, 02478. Tel: 617.855.2364. Email address: Shuang.Li@MGH.HARVARD.EDU) should be contacted if someone wants to request the data from this study.

\section{Authors' contributions}

SL, RM, SS contributed to the concept and design of study. CM, VV, BB collected the data. DD and SL analyzed data. DD, CM, VV, BB, SL, RM, SL wrote and revised the manuscript. All authors read and approved the final manuscripts.

\section{Acknowledgements}

Not applicable.

\section{Ethics approval and consent to participate}

This retrospective data analysis was approved by the Institutional Review Board (IRB) of Mass General Brigham. All methods were carried out in accordance with relevant guidelines and regulations. Consent to participate was waived per Institutional Review Board (IRB) of Mass General Brigham.

\section{Consent for publication}

Not applicable.

\section{Competing interests}


The authors declare that they have no competing interests.

\section{References}

1. Hasin DS, Sarvet AL, Meyers JL, Saha TD, Ruan WJ, Stohl M, et al. Epidemiology of Adult DSM-5 Major Depressive Disorder and Its Specifiers in the United States. JAMA Psychiatry. 2018;75.

2. World Health Organization. Depression and Other Common Mental Disorders: Global Health Estimates. https://apps.who.int/iris/bitstream/handle/10665/254610/WHO-MSD-MER-2017.2eng.pdf. 2017.

3. Mclntyre RS, Filteau M-J, Martin L, Patry S, Carvalho A, Cha DS, et al. Treatment-resistant depression: Definitions, review of the evidence, and algorithmic approach. J Affect Disord. 2014;156.

4. Berman RM, Cappiello A, Anand A, Oren DA, Heninger GR, Charney DS, et al. Antidepressant effects of ketamine in depressed patients. Biol Psychiatry. 2000;47.

5. Wilkinson ST, Sanacora G. KETAMINE: A POTENTIAL RAPID-ACTING ANTISUICIDAL AGENT? Depress Anxiety. 2016;33.

6. Andrade C. Oral Ketamine for Depression, 1: Pharmacologic Considerations and Clinical Evidence. J Clin Psychiatry. 2019;80. doi:10.4088/JCP.19f12820.

7. Domany Y, Bleich-Cohen M, Tarrasch R, Meidan R, Litvak-Lazar O, Stoppleman N, et al. Repeated oral ketamine for out-patient treatment of resistant depression: randomised, double-blind, placebocontrolled, proof-of-concept study. Br J Psychiatry. 2019;214.

8. Fava M, Freeman MP, Flynn M, Judge H, Hoeppner BB, Cusin C, et al. Correction: Double-blind, placebo-controlled, dose-ranging trial of intravenous ketamine as adjunctive therapy in treatmentresistant depression (TRD). Mol Psychiatry. 2020;25.

9. Marcantoni WS, Akoumba BS, Wassef M, Mayrand J, Lai H, Richard-Devantoy S, et al. A systematic review and meta-analysis of the efficacy of intravenous ketamine infusion for treatment resistant depression: January 2009 - January 2019. J Affect Disord. 2020;277.

10. Caddy C, Amit BH, McCloud TL, Rendell JM, Furukawa TA, McShane R, et al. Ketamine and other glutamate receptor modulators for depression in adults. Cochrane Database Syst Rev. 2015.

11. Fond G, Loundou A, Rabu C, Macgregor A, Lançon C, Brittner M, et al. Ketamine administration in depressive disorders: a systematic review and meta-analysis. Psychopharmacology (Berl). 2014;231.

12. Han Y, Chen J, Zou D, Zheng P, Li Q, Wang H, et al. Efficacy of ketamine in the rapid treatment of major depressive disorder: a meta-analysis of randomized, double-blind, placebo-controlled studies. Neuropsychiatr Dis Treat. 2016;Volume 12.

13. McGirr A, Berlim MT, Bond DJ, Fleck MP, Yatham LN, Lam RW. A systematic review and meta-analysis of randomized, double-blind, placebo-controlled trials of ketamine in the rapid treatment of major depressive episodes. Psychol Med. 2015;45.

14. Xu Y, Hackett M, Carter G, Loo C, Gálvez V, Glozier N, et al. Effects of Low-Dose and Very Low-Dose Ketamine among Patients with Major Depression: a Systematic Review and Meta-Analysis. Int $J$ 
Neuropsychopharmacol. 2016;19.

15. Andrade C. Ketamine for Depression, 4: In What Dose, at What Rate, by What Route, for How Long, and at What Frequency? J Clin Psychiatry. 2017;78.

16. Malhi GS, Byrow Y, Cassidy F, Cipriani A, Demyttenaere K, Frye MA, et al. Ketamine: stimulating antidepressant treatment? BJPsych Open. 2016;2.

17. Vande Voort JL, Morgan RJ, Kung S, Rasmussen KG, Rico J, Palmer BA, et al. Continuation phase intravenous ketamine in adults with treatment-resistant depression. J Affect Disord. 2016;206.

18. Zuckerman H, Pan Z, Park C, Brietzke E, Musial N, Shariq AS, et al. Recognition and Treatment of Cognitive Dysfunction in Major Depressive Disorder. Front Psychiatry. 2018;9.

19. Morgan CJA, Curran HV. Acute and chronic effects of ketamine upon human memory: a review. Psychopharmacology (Berl). 2006;188.

20. Chan KWS, Lee TMC, Siu AMH, Wong DPL, Kam C-M, Tsang SKM, et al. Effects of chronic ketamine use on frontal and medial temporal cognition. Addict Behav. 2013;38.

21. Morgan CJA, Dodds CM, Furby H, Pepper F, Fam J, Freeman TP, et al. Long-Term Heavy Ketamine Use is Associated with Spatial Memory Impairment and Altered Hippocampal Activation. Front Psychiatry. 2014;5.

22. Morgan CJA, Muetzelfeldt L, Curran HV. Consequences of chronic ketamine self-administration upon neurocognitive function and psychological wellbeing: a 1-year longitudinal study. Addiction. 2010;105.

23. Hayley A, Green M, Downey L, Keane M, Kostakis P, Shehabi Y. Neurocognitive and behavioural performance of healthy volunteers receiving an increasing analgesic-range infusion of ketamine. Psychopharmacology (Berl). 2018;235.

24. Morgan CJA, Mofeez A, Brandner B, Bromley L, Curran HV. Acute Effects of Ketamine on Memory Systems and Psychotic Symptoms in Healthy Volunteers. Neuropsychopharmacology. 2004;29.

25. Zheng W, Zhou Y-L, Liu W-J, Wang C-Y, Zhan Y-N, Li H-Q, et al. Neurocognitive performance and repeated-dose intravenous ketamine in major depressive disorder. J Affect Disord. 2019;246.

26. Zhou Y, Zheng W, Liu W, Wang C, Zhan Y, Li H, et al. Neurocognitive effects of six ketamine infusions and the association with antidepressant response in patients with unipolar and bipolar depression. $J$ Psychopharmacol. 2018;32.

27. Chen M-H, Li C-T, Lin W-C, Hong C-J, Tu P-C, Bai Y-M, et al. Cognitive function of patients with treatment-resistant depression after a single low dose of ketamine infusion. J Affect Disord. 2018;241.

28. Murrough JW, Wan L-B, lacoviello B, Collins KA, Solon C, Glicksberg B, et al. Neurocognitive effects of ketamine in treatment-resistant major depression: association with antidepressant response. Psychopharmacology (Berl). 2014;231.

29. Shiroma PR, Albott CS, Johns B, Thuras P, Wels J, Lim KO. Neurocognitive performance and serial intravenous subanesthetic ketamine in treatment-resistant depression. Int $\mathrm{J}$ Neuropsychopharmacol. 
2014;17.

30. Basso L, Bönke L, Aust S, Gärtner M, Heuser-Collier I, Otte C, et al. Antidepressant and neurocognitive effects of serial ketamine administration versus ECT in depressed patients. J Psychiatr Res. 2020;123.

31. Rush AJ, Trivedi MH, Ibrahim HM, Carmody TJ, Arnow B, Klein DN, et al. The 16-Item quick inventory of depressive symptomatology (QIDS), clinician rating (QIDS-C), and self-report (QIDS-SR): a psychometric evaluation in patients with chronic major depression. Biol Psychiatry. 2003;54.

32. Nasreddine ZS, Phillips NA, BÃ@dirian V, Charbonneau S, Whitehead V, Collin I, et al. The Montreal Cognitive Assessment, MoCA: A Brief Screening Tool For Mild Cognitive Impairment. J Am Geriatr Soc. 2005;53.

33. Loo C. Can we confidently use ketamine as a clinical treatment for depression? The Lancet Psychiatry. 2018;5.

34. Fedgchin M, Trivedi M, Daly EJ, Melkote R, Lane R, Lim P, et al. Efficacy and Safety of Fixed-Dose Esketamine Nasal Spray Combined With a New Oral Antidepressant in Treatment-Resistant Depression: Results of a Randomized, Double-Blind, Active-Controlled Study (TRANSFORM-1). Int J Neuropsychopharmacol. 2019;22.

35. Sanacora G, Frye MA, McDonald W, Mathew SJ, Turner MS, Schatzberg AF, et al. A Consensus Statement on the Use of Ketamine in the Treatment of Mood Disorders. JAMA Psychiatry. 2017;74.

36. Singh JB, Fedgchin M, Daly EJ, De Boer P, Cooper K, Lim P, et al. A Double-Blind, Randomized, Placebo-Controlled, Dose-Frequency Study of Intravenous Ketamine in Patients With TreatmentResistant Depression. Am J Psychiatry. 2016;173.

37. Fava M, Freeman MP, Flynn M, Judge H, Hoeppner BB, Cusin C, et al. Double-blind, placebocontrolled, dose-ranging trial of intravenous ketamine as adjunctive therapy in treatment-resistant depression (TRD). Mol Psychiatry. 2020;25.

38. Aan Het Rot M, Zarate Jr. CA, Charney DS, Mathew SJ. Ketamine for depression: where do we go from here? Biol Psychiatry. 2012;72:537-47. doi:10.1016/j.biopsych.2012.05.003.

39. Grunebaum MF, Galfalvy HC, Choo T-H, Keilp JG, Moitra VK, Parris MS, et al. Ketamine for Rapid Reduction of Suicidal Thoughts in Major Depression: A Midazolam-Controlled Randomized Clinical Trial. Am J Psychiatry. 2018;175.

40. Ding R, Li Y, Du A, Yu H, He B, Shen R, et al. Changes in hippocampal AMPA receptors and cognitive impairments in chronic ketamine addiction models: another understanding of ketamine CNS toxicity. Sci Rep. 2016;6.

41. Ke X, Ding $Y, X u K, H e H$, Wang D, Deng $X$, et al. The profile of cognitive impairments in chronic ketamine users. Psychiatry Res. 2018;266.

\section{Figures}




\section{Effects of repeated ketamine infusions on cognition}

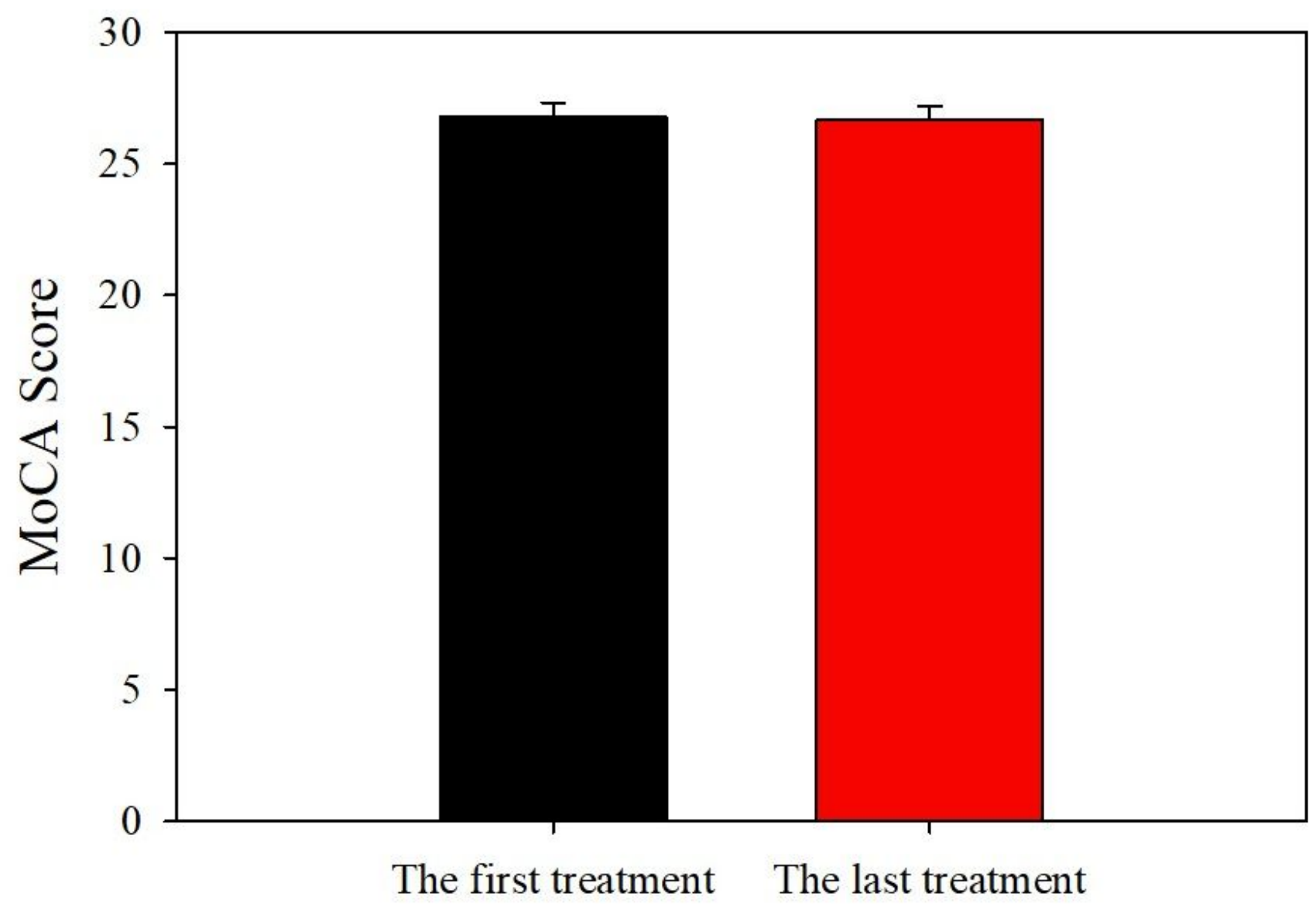

Figure 1

The effect of repeated ketamine infusions on cognition. The data are expressed as mean+S.E.M. There was no significance of difference between two groups: $P>0.05$, (paired t-test). 


\section{Effects of repeated ketamine infusions on depression}

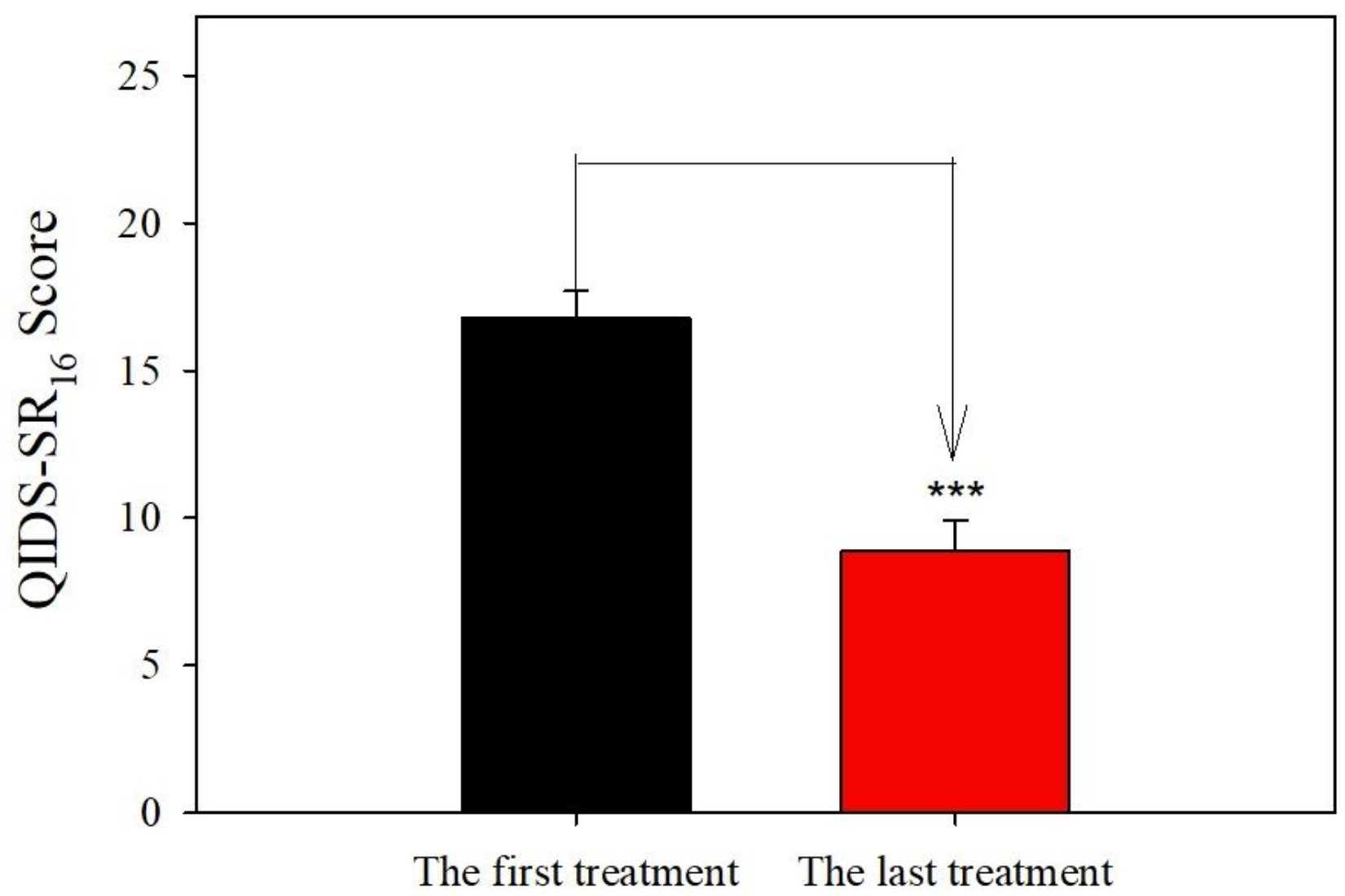

Figure 2

The effect of repeated ketamine infusions on depression. The data are expressed as mean+S.E.M. Significance of difference: ${ }^{\star} * \star P<, 0.001$, (paired t-test). 


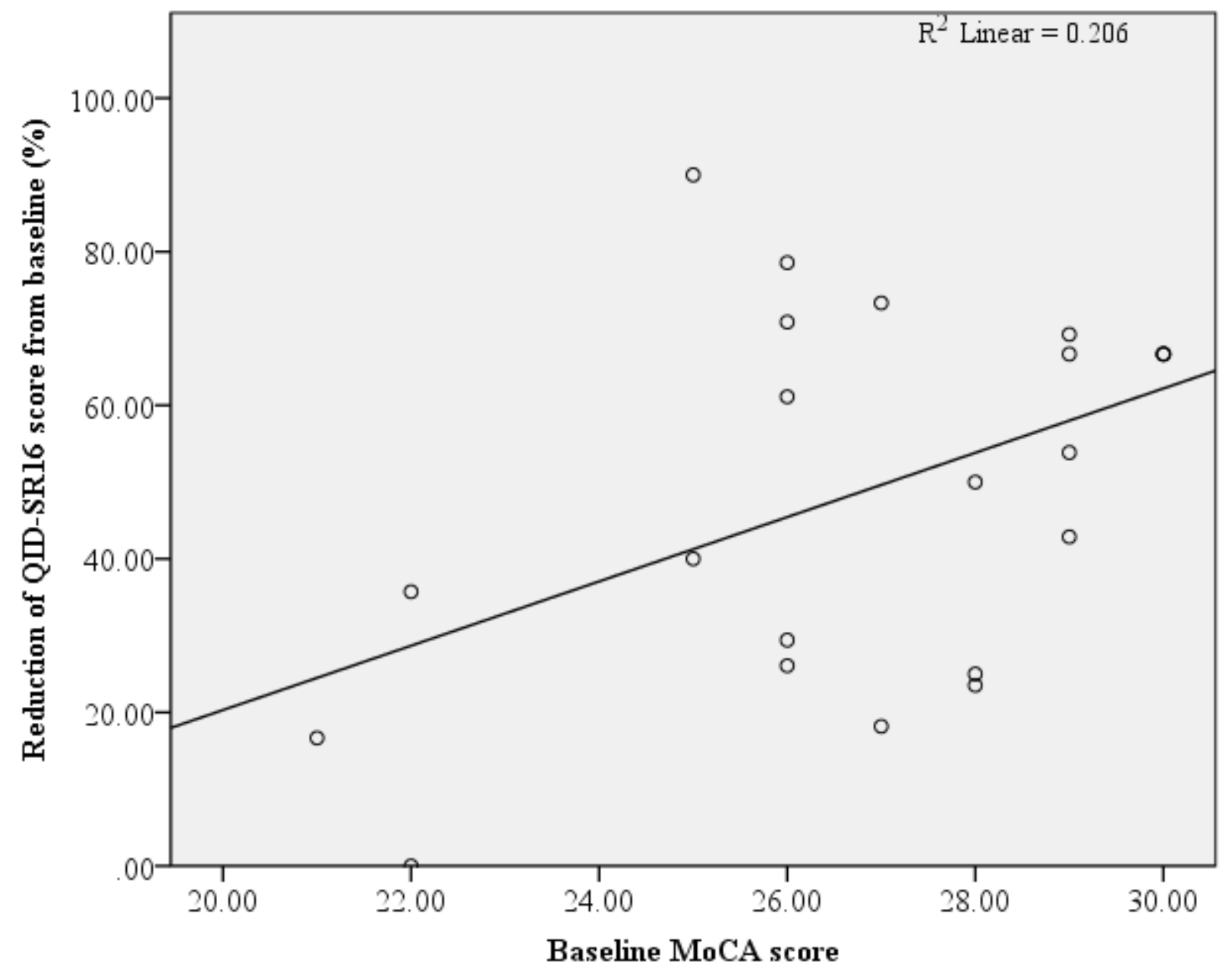

Figure 3

The scatter plot of linear regression of MoCA score and reduction (\%) of QIDS-SR16 from baseline. 